\title{
Endoscopic Adenoidectomy in Children With Otitis Media With Effusion and Mild Hearing Loss
}

\author{
Pasquale Capaccio $^{1} \cdot$ Sara Torretta $^{2}$ - Givlia Anna Marciante ${ }^{2} \cdot$ Paola Marchisio $^{3} \cdot$ Stella Forti $^{1} \cdot$ Lorenzo Pignataro $^{2}$ \\ ${ }^{1}$ Department of Biomedical Surgical and Dental Sciences, ${ }^{2}$ Department of Clinical Sciences and Community Health, and ${ }^{3}$ Department of \\ Physiopathology and Transplantation, Fondazione IRCCS Ca 'Granda Ospedale Maggiore Policlinico,
}

Universita`degli Studi di Milano, Milan, Italy

Objectives. Surgical management of children with chronic otitis media with effusion (OME) includes tympanostomy tube insertion or adenoidectomy, alone or with myringotomy and tube insertion. The aim of this study was to compare the effectiveness of transoral microdebrider endoscopic-assisted adenoidectomy (TOMEA) and traditional adenoidectomy in the management of children with mild hearing loss due to OME and chronic adenoiditis.

Methods. This prospective, double-blind and controlled study involved 120 consecutive patients aged 4-12 years, who were randomised 1:1 to undergo TOMEA or traditional adenoidectomy under general anesthesia. All the patients underwent a complete otolaryngological examination, including nasopharyngeal fibre endoscopy (NFE), pneumatic otoscopy, otomicroscopy, tympanometry and supraliminar tonal audiometry, upon enrolment, and three and nine months postoperatively.

Results. There were no statistically significant differences in age or gender distribution between the TOMEA group (mean age, $4.9 \pm 1.1$ years; $53.3 \%$ males) and the traditional adenoidectomy group (mean age, $5.3 \pm 0.9$ years; $56.7 \%$ males). Both procedures led to a significant improvement in choanal patency $(P<0.01)$ and all of the otological and audiological parameters $(P<0.01) 3$ and 9 months postoperatively, although postoperative NFE showed that the mean percentage of residual choanal obstruction was significantly less in the TOMEA group $(P=0.02)$. There was no significant between-group difference in the percentage of children with tympanic membrane changes, but the postoperative prevalence of children with a type B tympanogram was significantly lower in the TOMEA group after 3 $(15.0 \%$ vs. $31.7 \%, P=0.05)$ and 9 months $(18.3 \%$ vs. $38.3 \%, P=0.02)$, as was the percentage of children with mild conductive hearing loss ( $3.3 \%$ vs. $23.3 \%, P<0.01$; and $8.3 \%$ vs. $28.3 \%, P<0.01)$.

Conclusion. Although both TOMEA and traditional adenoidectomy are effective in treating children with mild hearing loss due to adenoidal hypertrophy and OME, the former achieves the greater reduction in residual adenoidal hypertrophy and better audiological outcomes.

Keywords. Otitis Media; Adenoids; Adenoidectomy; Hearing Loss

\section{INTRODUCTION}

Otitis media with effusion (OME) is an inflammatory disorder

\footnotetext{
- Received March 8, 2015

Revised April 13, 2015

Accepted April 28, 2015

- Corresponding author: Pasquale Capaccio

Department of Biomedical Surgical and Dental Sciences, Fondazione

IRCCS Ca` Granda Ospedale Maggiore Policlinico, Universita` degli Studi di Milano,Via F. Sforza 35, 20122 Milano, Italy

Tel: +39-255032563, Fax: +39-250320248

E-mail: pasquale.capaccio@unimi.it
}

of the middle ear that is characterised by the presence of endotympanic fluid without any sign or symptom of acute ear infection [1], which may lead to hearing loss (HL) or long-term sequelae and have a negative impact on speech development and behaviour [1-3]. It is estimated that up to $90 \%$ of children experience at least one episode before reaching school age $(60 \%$ of the episodes occur in the first 2 years of life) [1,2], and that a total of 2.2 million pediatric episodes a year are diagnosed in the United States alone.

Given the low rate of spontaneous resolutions $(<30 \%$ in children aged $<4$ years) [3] and the ineffectiveness of medical treat-

Copyright @ 2016 by Korean Society of Otorhinolaryngology-Head and Neck Surgery.

This is an open-access article distributed under the terms of the Creative Commons Attribution Non-Commercial License (http://creativecommons.org/licenses/by-nc/4.0)

which permits unrestricted non-commercial use, distribution, and reproduction in any medium, provided the original work is properly cited. 
ment, the recommended treatment is still surgery, which may take the form of tympanostomy tube insertion or adenoidectomy with or without myringotomy or tube insertion [3-5].Adenoidectomy alone should be considered in children with OME associated with postnasal obstruction, chronic rhinosinusitis or adenoiditis, or those who have previously undergone repeat surgery for $\mathrm{OME}$, and has been reported to be most beneficial in children aged $>3$ years regardless of adenoid size [5]. Adenoidal hypertrophy and the closeness of the adenoids to the ostium of the Eustachian tube seem to be the most important factors in the pathogenesis of OME: we have recently found that adenoidal bacterial biofilms (recognised causes of OME) are significantly more prevalent near the ostium, thus suggesting that the adenoids are a reservoir for biofilm-producing bacteria that can spread through the Eustachian tube to colonise the middle ear [6].

Under these conditions, the aim of surgery in patients with OME and adenoidal hypertrophy should be the complete removal of the adenoids not only in order to restore Eustachian tube patency, but also to ensure the total eradication of bacterial biofilms. Adenoidectomy can be performed using the traditional blind approach or an endoscopic-assisted technique that allows the more precise and selective removal of lymphoid tissue under direct inspection in order to avoid the persistence of small adenoidal residues near the ostium of the Eustachian tube.

The aim of this study was to compare the effectiveness of transoral microdebrider endoscopic-assisted adenoidectomy (TOMEA) and traditional adenoidectomy in the management of children with mild HL due to OME and chronic adenoiditis.

\section{MATERIALS AND METHODS}

\section{Study design and setting}

This randomised controlled trial was carried out at the University of Milan's Department of Clinical Sciences and Community Health and Department of Physiopathology and Transplantation between January 2012 and January 2015. The protocol was approved by our local Ethics Committee, and written informed consent was obtained from the children's parents or legal guardians.

\section{Study subjects}

The inclusion criteria were an age of 4-12 years, and the presence of chronic adenoiditis and OME (middle ear effusion without any sign of acute concomitant middle ear inflammation for at least 3 months, documented by means of pneumatic otoscopy and timpanometry) with mild conductive HL (mean threshold $<40 \mathrm{~dB}$ at air conduction thresholds of 500, 1,000, 2,000, and $4,000 \mathrm{~Hz}$ ) [7]. Chronic adenoiditis was defined as adenoidal hypertrophy (grade III or more according to Cassano's classification, as assessed by means of flexible nasopharyngeal endoscopy) [8] with ongoing nasopharyngeal inflammation/infection (i.e., at least 3 episodes of acute adenoiditis requiring antibiotic therapy in 6 months, or at least 4 episodes in 12 months), with persistent mucous secretion on the adenoidal surface [9].

The exclusion criteria were concomitant systemic diseases; craniofacial, neuromuscular, immunological, syndromic or defined genetic abnormalities; allergy (documented within the previous 12 months by positive skin prick tests or radio-allergosorbent tests and serum IgE levels); chronic eardrum perforation; previous ear surgery or adenoidectomy; moderate-to-severe HL ( $\geq 40 \mathrm{~dB}$ ) [7]; and upper respiratory tract infection or antibiotic therapy in the previous 14 days.

\section{Assessments}

Upon enrolment, and 3 and 9 months postoperatively, all of the children underwent pediatric and otolaryngological evaluations, including:

- Pneumatic otoscopy and otomicroscopy aimed at evaluating the integrity, colour, mobility, position, lightening and translucency of the tympanic membrane, and detecting any signs of acute middle ear inflammation. Impaired otoscopy was defined as the presence of changes of the tympanic membrane such as fibrotic scars, tympanosclerosis, variable opacity, abnormal airfluid levels, and retraction pockets.

-Tympanometry, which was used to confirm middle ear effusion on the basis of the presence of a type B tympanogram [10].

- Hearing tests (i.e., conditioned play audiometry in children aged $<5$ years or pure tone audiometry in children aged $\geq 6$ years) conducted by an experienced audiologist [7].

- Flexible nasopharyngeal fibre endoscopy (NFE) (Pentax FNL7RP3, Pentax S.r.l., Milan, Italy) performed by two experienced otolaryngologists as previously described [11].

Upon enrolment, the patients were randomised 1:1 to undergo TOMEA (group A) or traditional adenoidectomy (group B) under general anesthesia. All of the surgical procedures were performed by two experienced otolaryngologists ( $\mathrm{PC}$ and LP).

\section{Surgical techniques}

After placing the patients in the Rose position, a Crowe-Davis mouth gag was used to splint the mouth open, and two small Nélaton catheters were passed through the patient's nose and brought through the mouth in order to retract the soft palate and increase the surgical view.

In group $\mathrm{A}$, both the $70^{\circ}$ endoscope with a video attachment and the microdebrider (a serrated, 4-mm diameter Diego-powered dissector adenoid blade, Gyrus ACMI, Hamburg, Germany) were inserted through the oral cavity. Surgical debridement started from the lowest vegetation on the posterior wall of the pharynx and ended with the upper choanal tissue, including the peritubaric region, and hemostasis was obtained by means of selective bipolar cauterisation (Fig. 1).

In group $\mathrm{B}$, adenoidectomy was performed by inserting a La Force adenotome through the mouth and blindly removing the nasopharyngeal adenoidal tissue; hemostasis was obtained by 
placing a package for some minutes.

Children were followed up for 9 months. In this interval only antibiotic treatment for acute infections were allowed. No treatment for OME was allowed.

\section{Statistical analysis}

The sample size was determined on the basis of published data regarding the efficacy of tube insertion in reducing the prevalence

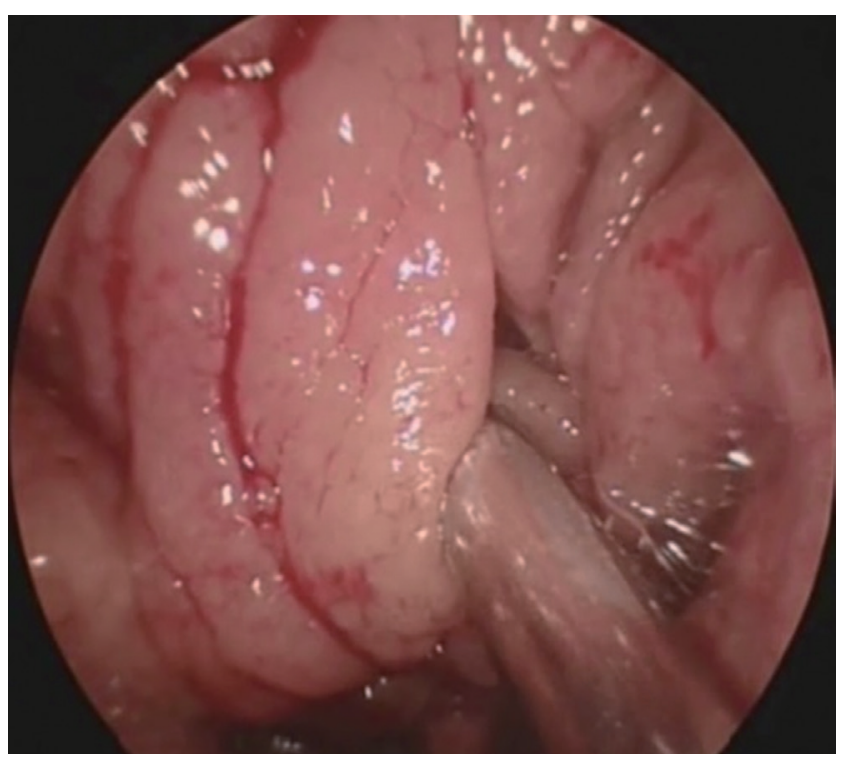

Fig. 1. Intraoperative screenshot during transoral microdebrider endoscopic-assisted adenoidectomy. of middle ear effusion in children with chronic OME [12]. Assuming that there would be no patients lost to follow-up and a standard deviation of 0.20 , it was calculated that 60 subjects in each group would lead to a beta error margin of 0.20 , an alpha value of 0.05 , and a power of $80 \%$.

The results are given as arithmetical mean values \pm standard deviation. Age distribution and percentage choanal obstruction due to adenoidal hypertrophy before and after surgery were tested using the Mann-Whitney nonparametric test, and Fisher exact probability test was used to analyse the between-group differences in the tympanometric, audiometric and otoscopic findings.

The statistical analyses were made using the IBM SPSS ver. 18.0 (IBM Co., Armonk, NY, USA); a $P$-value of <0.05 was considered statistically significant.

\section{RESULTS}

There were no statistically significant differences in age or gender distribution between the TOMEA group (mean age, $4.9 \pm 1.1$ years; $53.3 \%$ males) and the traditional adenoidectomy group (mean age, $5.3 \pm 0.9$ years; $56.7 \%$ males) (Table 1 ).

Both procedures led to a significant improvement in choanal patency $(P=0.01)$ and all of the otological parameters compared to the preoperative status, as shown by the significant reduction $(P<0.01)$ in the percentage of children with tympanic membrane changes, a type B tympanogram, and conductive HL 3 and 9

Table 1. Demographic and clinical characteristics of patients undergo transoral microdebrider endoscopic-assisted adenoidectomy (group A) and traditional adenoidectomy (group B)

\begin{tabular}{|c|c|c|c|c|c|c|}
\hline Parameter & Group & Preop. & 3 Months postop. & $P$-value* & 9 Months postop. & $P$-value \\
\hline \multirow[t]{3}{*}{ Male sex $(n=60)$} & $A$ & $32(53.3)$ & - & - & - & - \\
\hline & B & $34(56.7)$ & - & - & - & - \\
\hline & $P$-value (group A vs. B) & NS & - & - & - & - \\
\hline \multirow[t]{3}{*}{ Age (yr) } & A & $4.9 \pm 1.1$ & - & - & - & - \\
\hline & B & $5.3 \pm 0.9$ & - & - & - & - \\
\hline & $P$-value (group A vs. B) & NS & - & - & - & - \\
\hline \multirow[t]{3}{*}{ Choanal obstruction (\%) } & A & $80.0 \pm 15.4$ & $5.5 \pm 7.5$ & $<0.01$ & $8.2 \pm 6.8$ & $<0.01$ \\
\hline & B & $75.8 \pm 14.9$ & $20.3 \pm 10.7$ & $<0.01$ & $26.4 \pm 7.5$ & $<0.01$ \\
\hline & $P$-value (group A vs. B) & NS & 0.02 & & 0.02 & \\
\hline \multirow[t]{3}{*}{ Impaired otoscopy $(n=60)$} & A & $60(100)$ & $14(23.3)$ & $<0.01$ & $17(28.3)$ & $<0.01$ \\
\hline & B & $60(100)$ & $17(28.3)$ & $<0.01$ & $19(31.7)$ & $<0.01$ \\
\hline & $P$-value (group A vs. B) & NS & NS & & NS & \\
\hline \multirow[t]{3}{*}{ Type B tympanogram $(n=60)$} & A & $60(100)$ & $9(15.0)$ & $<0.01$ & $11(18.3)$ & $<0.01$ \\
\hline & B & $60(100)$ & $19(31.7)$ & $<0.01$ & $23(28.3)$ & $<0.01$ \\
\hline & $P$-value (group A vs. B) & NS & 0.05 & & 0.02 & \\
\hline \multirow[t]{3}{*}{ Hearing loss $(n=60)$} & A & $60(100)$ & $2(3.3)$ & $<0.01$ & $5(8.3)$ & $<0.01$ \\
\hline & B & $60(100)$ & $14(23.3)$ & $<0.01$ & $17(28.3)$ & $<0.01$ \\
\hline & $P$-value (group A vs. B) & NS & $<0.01$ & & $<0.01$ & \\
\hline
\end{tabular}

Values are presented as number (\%) or mean \pm standard deviation.

Preop., preoperative; postop., postoperative; NS, nonsignificant.

*Preop. vs. 6 months postop. ${ }^{\dagger}$ Preop. vs. 9 months postop. 
months postoperatively.

However, postoperative NFE showed that the postoperative mean percentage of residual choanal obstruction was lower in group A after both $3(5.5 \% \pm 7.5 \%$ vs. $20.3 \% \pm 10.7 \%, P=0.02)$ and 9 months $(8.2 \% \pm 6.8 \%$ vs. $26.4 \% \pm 7.5 \%, P=0.02)$. There was no significant between-group difference in the percentage of children with tympanic membrane changes $3(23.3 \%$ vs. $28.3 \%)$ and 9 months after surgery (18.3\% vs. $31.7 \%)$, but the percentage of children with a type B tympanogram was significantly lower in group A at both timepoints $(15.0 \%$ vs. $31.7 \%$, $P=0.05$; and $18.3 \%$ vs. $38.3 \%, P=0.02$ ), as was the percentage of children with mild conductive HL (3.3\% vs. $23.3 \%, P<0.01$; and $8.3 \%$ vs. $28.3 \%, P<0.01$ ).

Antibiotic treatment for acute respiratory infections was administered in few children in 7 out of 60 children in group A $(11.7 \%)$ and in 9 out of 60 children in group B $(15.0 \%)(P=$ not significant). No episodes of acute otitis media were reported.

\section{DISCUSSION}

Adenoidectomy is one of the therapeutic procedures used to manage OME, and various surgical techniques have been proposed in attempt to improve clinical outcomes [13,14]. There is still a lot of controversy regarding the role of adenoidectomy in reducing the incidence of OME. In a recent Cochrane review Van den Aardweg et al. [15] reported that is significant benefit of adenoidectomy in the resolution of middle ear effusion in children with OME, but the benefit to hearing is small and the effects on changes in the tympanic membrane are unknown. Therefore, the risks of the surgical procedure should be balanced with the potential benefits in making a decision for or against this surgery. In addition to this, the indications of guidelines in different countries are quite different, ranging from tubes as first choice and adenoidectomy as adjuvant only in cases with adenoidal hypertrophy $[16,17]$ to adenoidectomy as first choice as suggested by Italian Guidelines [18]. In our population miringotomy was not performed, not tubes were inserted. We followed the Italian Guidelines on the appropriateness of tonsillectomy and adenoidectomy [18] which states that adenoidectomy is indicated in children with chronic OME with hypertrophic adenoids obstructing the Eustachian tube opening or chronic adenoiditis.

It is well known that both traditional and endoscopic adenoidectomy reduce hypertrophic adenoid tissue $[13,14]$ but, to the best of our knowledge, their effects on otological and nasal outcomes in patients with long-lasting middle ear effusion and adenoidal hypertrophy have not been previously compared. The aim of this study was to begin filling this gap.

Tympanometric evidence of the resolution of OME was observed 9 months postoperatively in most of the patients who underwent TOMEA and in more than $60 \%$ of those who underwent traditional adenoidectomy. In particular, type B tympano- gram suggesting complete middle ear effusion [10] was more frequently in children belonging to group $\mathrm{B}$ compared to those belonging to group A ( $38.3 \%$ vs. $18.3 \%, P=0.02)$ at the end of follow-up, while type $C$ tympanogram suggesting Eustachian tube dysfunction was reported in 12 out of 60 children $(20.0 \%)$ in group A and in 14 out of 60 children $(23.3 \%)$ in group B ( $P=$ not significant) 9 months after surgery. These data suggest that adenoidectomy globally improve middle ear ventilation and drainage, but complete recovery of Eustachian tube function more frequently occurred afterTOMEA.

Both procedures also reduced HL as assessed by means of audiological tests 3 and 9 months after surgery, but the reduction was greater in the patients undergoing TOMEA. The superiority of adenoidectomy over other treatments for OME has so far been unclear because, although some authors have previously shown that adenoidectomy is more effective than no adenoidectomy in terms of tympanometric recovery and hearing levels, this has not been confirmed by others [19-22]. However, our findings provide the first evidence indicating that TOMEA is more effective in treating OME-related HL than traditional adenoidectomy in the medium term.

Nasal fibre endoscopy at the end of the follow-up showed that TOMEA also led to significantly less mean residual postnasal obstruction in comparison with traditional adenoidectomy ( $8 \%$ vs. $26 \%$ ). It has been reported that the endoscopic adenoidectomy is effective in reducing long-term postnasal obstruction due to hypertrophic adenoids [23,24], but the percentages of residual postnasal and peritubaric obstruction described after traditional adenoidectomy vary; nevertheless, our findings concerning the residual nasopharyngeal obstruction obtained using the two surgical techniques are in line with other surgical experiences [23,24].

It is clear that the more complete surgical removal of excess nasopharyngeal tissue (especially in the peritubaric area) usually allowed by endoscopic surgery reduces the risk of peritubaric and velopharyngeal muscle damage [25], and that restoration of the patency of the torus tubarius facilitates middle ear drainage and ventilation. Ark et al. [26] have found that residual adenoids could be detected near at least one torus tubarius in about $11.4 \%$ of their patients who had undergone traditional adenoidectomy, and at the velopharyngeal port in $6.3 \%$, and the partial residual obstruction of the torus tubarius caused by this excess tissue may explain the persistence of middle ear effusion in such patients. Although OME is often caused by impaired Eustachian tube patency due to obstructive hypertrophic adenoids, it may also be caused by infected adenoids: it has been recently reported that the nasopharynx plays a special role in pediatric pneumococcal colonisation as the primary reservoir of pneumococci (which are frequently involved in middle ear inflammation) [27], and so adenoidectomy may therefore affect the rate of nasopharyngeal pneumococcal carriage [28].

It has also been recently reported that middle ear effusion, 
which has historically been considered a sterile condition, is actually sustained by bacterial species that can only be detected by means of polymerase chain reaction and complex electron microscopy $[29,30]$. These bacteria, which are highly resistant to traditional antibiotics and immune system activity, quiescently colonise the middle ear at a low replication rate, but become periodically activated and deliver planktonic species known as bacterial biofilms. Nasopharyngeal bacterial biofilms are mainly produced by classical otopathogens chronically colonise adenoidal surfaces (particularly near the torus tubarius) in children with chronic adenoiditis and, during the delivery of the planktonic species responsible for periodic acute exacerbations, "metastasise" through the Eustachian tube to the middle ear, thus leading to chronic tubaric and middle ear mucosal infection [29]. This may explain the beneficial role of adenoidectomy in the therapeutic management of children who have previously undergone repeated myringotomies whose recurrent OME is due to the surgical debridement of the adenoidal bacterial biofilm reservoir regardless of the degree of obstruction [30].

A statistically significant reduction in the percentage of patients with tympanic membrane changes was observed in both groups three and nine months postoperatively, with no difference between the 2 groups. The persistence of impaired otoscopic signs such as fibrotic scars and variable opacity in some patients should not be considered a negative outcome as it should be related to the lasting of the effusion with a following reduced chance of regression. To the best of our knowledge, the long-term effects of adenoidectomy on such changes related to long-lasting OME has not been previously investigated, and our albeit encouraging results do not allow us to draw any definite conclusions.

These preliminary findings in a small study population suggest that both forms of adenoidectomy are effective in managing OME in patients with mild HL and adenoid hypertrophy, and may indicate that the mild HL associated with OME is reversible. However, the audiological and endoscopic results seem to favour TOMEA over traditional adenoidectomy, and it should be therefore considered in the therapeutic management of young patients with OME and adenoidal disease.

\section{CONFLICT OF INTEREST}

No potential conflict of interest relevant to this article was reported.

\section{REFERENCES}

1. Tos M. Epidemiology and natural history of secretory otitis. Am J Otol. 1984 Oct;5(6):459-62.

2. Casselbrant ML, Mandel EM. Epidemiology. In: Rosenfeld RM, Bluestone CD, editors. Evidence-based otitis media. 2nd ed. London: BC Decker; 2003. p. 147-62.
3. Rosenfeld RM, Kay D. Natural history of untreated otitis media. Laryngoscope. 2003 Oct;113(10):1645-57.

4. Rosenfeld RM, Schwartz SR, Pynnonen MA,Tunkel DE, Hussey HM, Fichera JS, et al. Clinical practice guideline: Tympanostomy tubes in children. Otolaryngol Head Neck Surg. 2013 Jul;149(1 Suppl):S1-35.

5. Paradise JL, Bluestone CD, Rogers KD, Taylor FH, Colborn DK, Bachman RZ, et al. Efficacy of adenoidectomy for recurrent otitis media in children previously treated with tympanostomy-tube placement. Results of parallel randomized and nonrandomized trials. JAMA. 1990 Apr;263:2066-73.

6. Torretta S, Drago L, Marchisio P, Gaffuri M, Clemente IA, Pignataro L. Topographic distribution of biofilm-producing bacteria in adenoid subsites of children with chronic or recurrent middle ear infections. Ann Otol Rhinol Laryngol. 2013 Feb;122(2):109-13.

7. Working group on manual pure-tone threshold audiometry. Guidelines for manual pure-tone threshold audiometry [Internet]. Rockville (MD): American Speech-Language-Hearing Association; c19972016 [cited 2015 Jan 15]. Available from: http://www.asha.org/policy/GL2005-00014.htm.

8. Cassano P, Gelardi M, Cassano M, Fiorella ML, Fiorella R. Adenoid tissue rhinopharyngeal obstruction grading based on fiberendoscopic findings: a novel approach to therapeutic management. Int J Pediatr Otorhinolaryngol. 2003 Dec;67(12):1303-9.

9. Marseglia GL, Poddighe D, Caimmi D, Marseglia A, Caimmi S, Ciprandi $\mathrm{G}$, et al. Role of adenoids and adenoiditis in children with allergy and otitis media. Curr Allergy Asthma Rep. 2009 Nov;9(6):460-4.

10. Onusko E.Tympanometry.Am Fam Physician. 2004 Nov;70(9):171320.

11. Torretta S, Marchisio P, Cappadona M, Baggi E, Pignataro L. Nasopharyngeal fiberendoscopy in children: a diagnostic challenge in current clinical practice: how we do it. Int J Pediatr Otorhinolaryngol. 2013 May;77(5):747-51.

12. Browning GG, Rovers MM, Williamson I, Lous J, Burton MJ. Grommets (ventilation tubes) for hearing loss associated with otitis media with effusion in children. Cochrane Database Syst Rev. 2010 Oct; (10):CD001801.

13. Feng Y, Yin S. Comparison of the powered-assisted adenoidectomy with adenoid curette adenoidectomy. Lin Chuang Er Bi Yan Hou Ke Za Zhi. 2006 Jan;20(2):54-7.

14. Bross-Soriano D, Schimelmitz-Idi J, Arrieta-Goez JR. Endoscopic adenoidectomy; use or abuse of the technology? Cir Cir. 2004 JanFeb;72(1):15-9.

15. van den Aardweg MT, Schilder AG, Herkert E, Boonacker CW, Rovers MM. Adenoidectomy for otitis media in children. Cochrane Database Syst Rev. 2010 Jan;(1):CD007810.

16. Robb PJ,Williamson I. Otitis media with effusion in children: current management. Paediatr Child Health (Oxford). 2012 Jan;22(1):9-12.

17. MRC Multicentre Otitis Media Study Group. Adjuvant adenoidectomy in persistent bilateral otitis media with effusion: hearing and revision surgery outcomes through 2 years in the TARGET randomised trial. Clin Otolaryngol. 2012 Apr;37(2):107-16.

18. National Guidelines System (SNLG); Italian National Institute of Health (ISS). Appropriateness and safety of tonsillectomy and/or adenoidectomy. Italian guidelines [Internet]. Rome: Italian National Institute of Health; 2008 [cited 2015 Apr 12]. Available from: http:// www.snlg-iss.it.

19. Boonacker CW, Rovers MM, Browning GG, Hoes AW, Schilder AG, Burton MJ. Adenoidectomy with or without grommets for children with otitis media: an individual patient data meta-analysis. Health Technol Assess. 2014 Jan;18(5):1-118.

20. Kujala T, Alho OP, Luotonen J, Kristo A, Uhari M, Renko M, et al. Tympanostomy with and without adenoidectomy for the prevention of recurrences of acute otitis media: a randomized controlled trial. Pediatr Infect Dis J. 2012 Jun;31(6):565-9. 
21. Wallace IF, Berkman ND, Lohr KN, Harrison MF, Kimple AJ, Steiner MJ. Surgical treatments for otitis media with effusion: a systematic review. Pediatrics. 2014 Feb;133(2):296-311.

22. Berkman ND, Wallace IF, Steiner MJ, Harrison M, Greenblatt AM, Lohr KN, et al. Otitis media with effusion: comparative effectiveness of treatments [Internet]. Rockville (MD): Agency for Healthcare Research and Quality (US); 2013 May [cited 2015 Jan 15]. Available from: http://www.ncbi.nlm.nih.gov/books/NBK143306/.

23. Saxby AJ, Chappel CA. Residual adenoid tissue post-curettage: role of nasopharyngoscopy in adenoidectomy. ANZ J Surg. 2009 Nov; 79(11):809-11.

24. Songu M,Altay C,Adibelli ZH, Adibelli H. Endoscopic-assisted versus curettage adenoidectomy: a prospective, randomized, doubleblind study with objective outcome measures. Laryngoscope. 2010 Sep;120(9):1895-9.

25. Havas T, Lowinger D. Obstructive adenoid tissue: an indication for powered-shaver adenoidectomy. Arch Otolaryngol Head Neck Surg. 2002 Jul;128(7):789-91.
26. Ark N, Kurtaran H, Ugur KS, Yilmaz T, Ozboduroglu AA, Mutlu C. Comparison of adenoidectomy methods: examining with digital palpation vs. visualizing the placement of the curette. Int J Pediatr Otorhinolaryngol. 2010 Jun;74(6):649-51.

27. Rapola S, Salo E, Kiiski P, Leinonen M, Takala AK. Comparison of four different sampling methods for detecting pharyngeal carriage of Streptococcus pneumoniae and Haemophilus influenzae in children. J Clin Microbiol. 1997 May;35(5):1077-9.

28. Mattila PS, Hammare-Malmi S, Saxen H, Kaijalainen T, Käyhty H, Tarkkanen J. Adenoidectomy and nasopharyngeal carriage of Streptococcus pneumoniae in young children. Arch Dis Child. 2010 Sep; 95(9):696-702.

29. Hall-Stoodley L, Hu FZ, Gieseke A, Nistico L, Nguyen D, Hayes J, et al. Direct detection of bacterial biofilms on the middle-ear mucosa of children with chronic otitis media. JAMA. 2006 Jul;296(2):202-11.

30. Saylam G, Tatar EC, Tatar I, Ozdek A, Korkmaz H. Association of adenoid surface biofilm formation and chronic otitis media with effusion. Arch Otolaryngol Head Neck Surg. 2010 Jun;136(6):550-5. 